ARTICLE

DOI: $10.1038 / s 41467-018-07498-y$

\title{
Efficient labeling and imaging of protein-coding genes in living cells using CRISPR-Tag
}

\author{
Baohui Chen ${ }^{1,2}$, Wei Zou ${ }^{3,4}$, Haiyue Xu1, Ying Liang ${ }^{1} \&$ Bo Huang (D) $^{5,6,7}$
}

The lack of efficient tools to image non-repetitive genes in living cells has limited our ability to explore the functional impact of the spatiotemporal dynamics of such genes. Here, we addressed this issue by developing a CRISPR-Tag system using one to four highly active sgRNAs to specifically label protein-coding genes with a high signal-to-noise ratio for visualization by wide-field fluorescence microscopy. Our approach involves assembling a CRISPR-Tag within the intron region of a fluorescent protein and then integrating this cassette to $\mathrm{N}$ - or $\mathrm{C}$-terminus of a specific gene, which enables simultaneous real-time imaging of protein and DNA of human protein-coding genes, such as HIST2H2BE, LMNA and HSPA8 in living cells. This CRISPR-Tag system, with a minimal size of $\sim 250$ bp DNA tag, represents an easily and broadly applicable technique to study the spatiotemporal organization of genomic elements in living cells.

\footnotetext{
${ }^{1}$ Department of Cell Biology, and Bone Marrow Transplantation Center of the First Affiliated Hospital, Zhejiang University School of Medicine, Zhejiang, Hangzhou 310058, China. ${ }^{2}$ Institute of Hematology, Zhejiang University \& Zhejiang Engineering Laboratory for Stem Cell and Immunotherapy, Hangzhou 310003, China. ${ }^{3}$ The Fourth Affiliated Hospital, Zhejiang University School of Medicine, Yiwu 322000, China. ${ }^{4}$ Insititute of Translational Medicine, Zhejiang University, Hangzhou 310058, China. ${ }^{5}$ Department of Pharmaceutical Chemistry, University of California, San Francisco, San Francisco 94143 CA, USA. ${ }^{6}$ Department of Biochemistry and Biophysics, University of California, San Francisco, San Francisco 94143 CA, USA. ${ }^{7}$ Chan Zuckerberg Biohub, San Francisco 94158 CA, USA. Correspondence and requests for materials should be addressed to B.C. (email: baohuichen@zju.edu.cn) or to B.H. (email: bo.huang@ucsf.edu)
} 
ndividual genes and genomic regions are located at different positions in the three-dimensional space of the nucleus ${ }^{1,2}$. The long-standing questions are whether the position of a gene affects its activity and how the gene positioning is maintained and regulated. There is no doubt that utilizing imaging techniques, which allow direct visualization of gene positioning and gene expression in living cells simultaneously, we will be able to uncover how gene position is linked to gene activity. Recent efforts toward this end focused on engineering a series of modular proteins with specific DNA recognition, including the clustered regularly interspaced short palindromic repeat (CRISPR)CRISPR-associated (Cas) system ${ }^{3-5}$. The catalytically dead version of Cas9 (dCas9) has been extensively explored for imaging endogenous genomic loci in living cells ${ }^{6,7}$. However, most of targets visualized by dCas 9 system are still limited to repetitive genomic region.

The major challenge is, when targeting non-repetitive genomic regions, it requires multiple sgRNAs function simultaneously to provide a sufficient signal-to-noise ratio for microscopy detection ${ }^{6}$. For example, to visualize a non-repetitive gene or regulatory element in mouse embryonic stem cells, 36 sgRNAs were expressed from three CARGO arrays to achieve efficient labeling 8 . Although two groups reported that the number of sgRNAs could be reduced to 3-4 using a combination of signal amplification and superresolution microscopy 9,10 , the labeling efficacy has not been quantitatively assessed. It is worth noting that signal amplification using multiple MS2 or PP7 repeats may introduce unspecific spots due to accumulation of nascent tagged sgRNA transcripts ${ }^{11}$.

It is a general issue for all CRISPR applications that the efficiency of Cas 9 targeting for any genomic locus can be dramatically influenced by the efficiency of sgRNAs used ${ }^{12}$. As such, it is very likely that only part of sgRNAs selected for DNA labeling function with high efficiency, which remains the major uncertainty of CRISPR-mediated genomic labeling. Thus, well-designed approaches using CRISPR imaging as readouts are critical to further optimize the DNA labeling system. Collectively, it is vital to achieve full potential of CRISPR imaging technology for labeling non-repetitive genomic elements. As such, we aim to develop DNA tags consisted of DNA sequence, which can be efficiently bound by dCas9-FP with highly active sgRNAs. In fluorescent repressor operator system (FROS), repeating sequences of $\mathrm{Lac}$ operator ( $\mathrm{LacO}, 256$ repeats) or Tet operator (TetO, 96 repeats) are used as DNA tags. Due to the large size and highly repetitive nature of $\mathrm{LacO} / \mathrm{TetO}$ array (usually $\sim 10$ and $\sim 4$ $\mathrm{kb}$, respectively) ${ }^{13,14}$, it remains technically challenging to use $\mathrm{LacO} / \mathrm{TetO}$ DNA tags to label a specific endogenous gene. Different from FROS system, DNA sequence recognized by dCas9FP is simply restricted by "NGG" PAM sequence. Therefore, we sought to assemble a shorter and more versatile DNA tag based on the CRISPR-Cas9 systems.

Here, we developed another type of DNA tags, termed "CRISPR-Tag", to label endogenous protein-coding genes in living cells. Two to six repeats of CRISPR targetable DNA sequences from Caenorhabditis elegans genome were integrated to a specific human gene and then imaged by fluorescent dCas9. We demonstrated a DNA tag knock-in strategy which allows simultaneous imaging of DNA and protein of the target gene. Most importantly, the use of CRISPR-Tags does not affect gene expression. All CRISPR-Tags we created are smaller than $850 \mathrm{bp}$. Thus, the CRISPR-Tag system represents an efficient, easy, and scalable DNA tagging system in living human cells.

\section{Results}

Assembly of CRISPR-Tag for labeling non-repetitive genes. CRISPR-Tag (diagram in Fig. 1a) was assembled with CRISPR- targeting sequences from $C$. elegans genome, which have been characterized for genome editing by several studies ${ }^{15-18}$. Six target sequences were picked according to the editing efficiency in worms and the on/off-target activity prediction by the web tool (http://crispr.mit.edu/). In addition, we generated a piece of artificial sequence based on the preference of nucleotides sequences that impact sgRNA efficacy ${ }^{19}$. In total, seven sgRNAs, termed sgTS1-sgTS7, were selected as the candidate sequences to assemble CRISPR-Tags (Supplementary Table 1). The first version of CRISPR-Tag (CRISPR-Tag_v1) contains six repeats. Four CRISPR-targeting sequences, TS1-TS4 were arranged in each repeat unit. Six repeat units were ligated to form a CRISPR-Tag using Golden Gate assembly. There are unique spacer sequences (25 bp) in between the repeats, which allows PCR or DNA sequencing to validate CRISPR-Tag sequence in cloning and knock-in experiments (Supplementary Fig. 1). To label a specific non-repetitive gene, we aim to first insert CRISPR-Tag into its $3^{\prime}$ UTR region or intron region by CRISPR knock-in, and then label the CRISPR-Tag with the nuclease-deficient Cas9 (dCas9) fused with fluorescent tags (Fig. 1a).

Signal amplification using tandem split GFP system. In order to minimize the size of the CRISPR-Tag, i.e. the number of target sequences, required to generate detectable signal, we adopted the tandem split GFP system to amplify the fluorescence from dCas $9^{20}$. To this end, we fused a repeating array containing 14 copies of GFP11 tags to dCas9 (dCas9-GFP ${ }_{14 \mathrm{x}}$ ), which can theoretically recruit as many as 14 copies of GFP (Supplementary Fig. 2a). When labeling the repeats in MUC4 and 5S rDNA genes and imaging with wide-field fluorescence microscopy, dCas9$\mathrm{GFP}_{14 \mathrm{x}}$ increased the signal-to-noise ratio (SNR) by a factor of 3 compared to dCas9-EGFP (Supplementary Fig. 2b, c), demonstrating an enhanced microscopy detection efficiency. However, when imaging non-repetitive regions in MUC4 gene with our previously published 36 sgRNAs that have not been individually validated, signal amplification alone by dCas9-GFP ${ }_{14 \mathrm{x}}$ still gives relatively low SNR. Only $\sim 26 \%$ of cells contain GFP-labeled spots due to low SNR (Supplementary Fig. 3). This result further indicates the need for a small set of well-validated sgRNAs as in our CRIPSR-Tag system.

CRISPR-Tag enables both DNA and protein labeling of genes. To demonstrate our CRISPR-Tag, we chose a protein-coding gene as our test system in order to simplify the selection process of CRISPR-Tag knock-in cells. Specifically, we inserted CRISPRTag into the $3^{\prime}$ UTR region of human HIST2H2BE (encoding histone H2B) by CRISPR knock-in using electroporation of Cas9/ sgRNA ribonucleoprotein complexes (RNPs) ${ }^{21}$ and double-cut plasmid donor ${ }^{22}$, which could increase targeting specificity and efficiency, respectively (diagram in Supplementary Fig. 4). We found that double-cut plasmid donor indeed resulted in higher knock-in efficiency than conventional plasmid donor and the efficiency highly dependents on cell types (Supplementary Fig. 5a, b). To select the integrated cells, we further added a mCherry sequence to the tag, which was knocked into the C-terminus of $\mathrm{H} 2 \mathrm{~B}$ as a FACS sorting marker (Supplementary Fig. 5c). Although most knock-in efficiencies were lower than $1 \%$, positive cells were successfully isolated by FACS sorting to generate stable CRISPR-Tag knock-in cell lines (Supplementary Table 2). CRISPR-Tag insertion was then validated by PCR and was further confirmed by the subcellular localization of $\mathrm{H} 2 \mathrm{~B}$-mCherry (Supplementary Figs. 5e and 6).

We then performed CRISPR imaging experiments using dCas9-GFP ${ }_{14 x}$ Tag. As expected, one bright puncta, representing H2B locus, was clearly visible upon transfection of four sgRNAs 
a

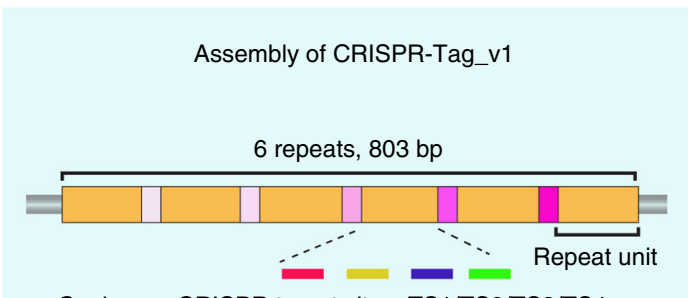

C. elegans CRISPR target sites: TS1/TS2/TS3/TS4

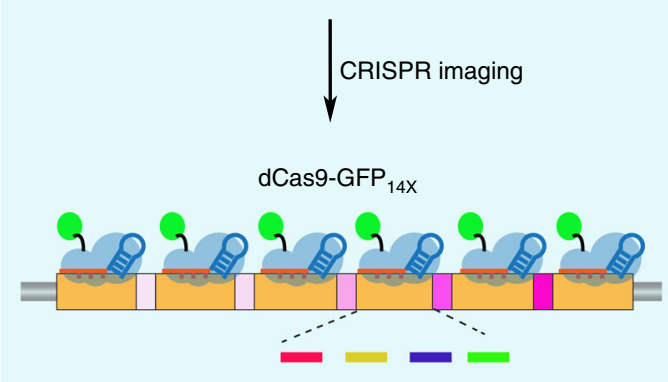

1-4 sgRNAs: sgTS1/sgTS2/sgTS3/sgTS4

b

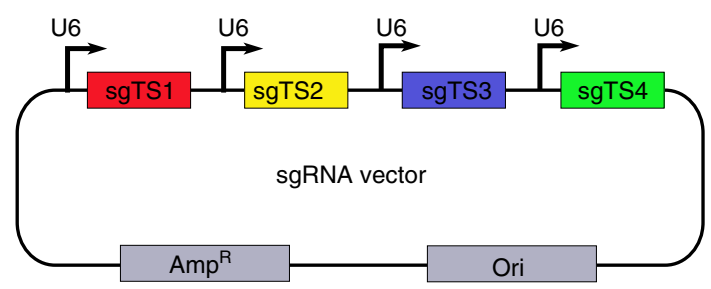

$\mathbf{f}$

CRISPR-Tag_v1

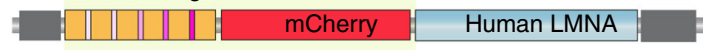
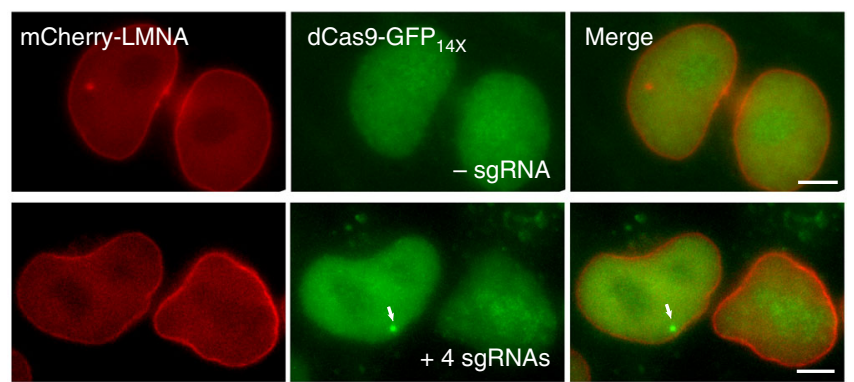

C

CRISPR-Tag in UTR CRISPR-Tag_v1
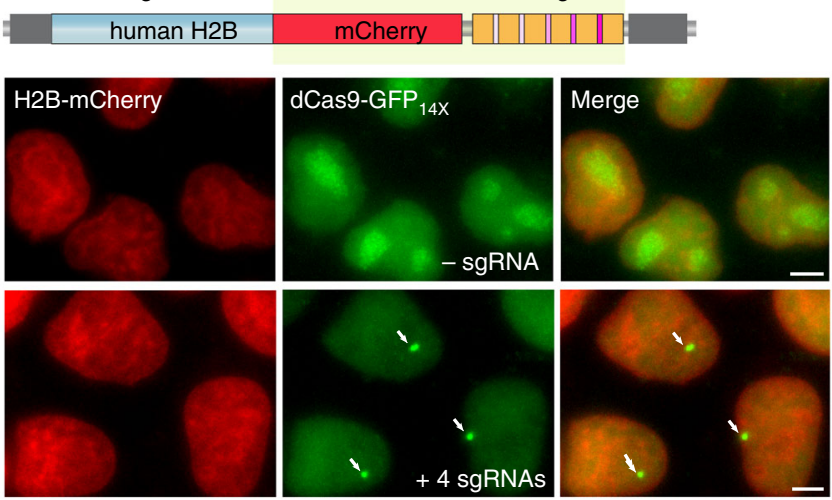

d
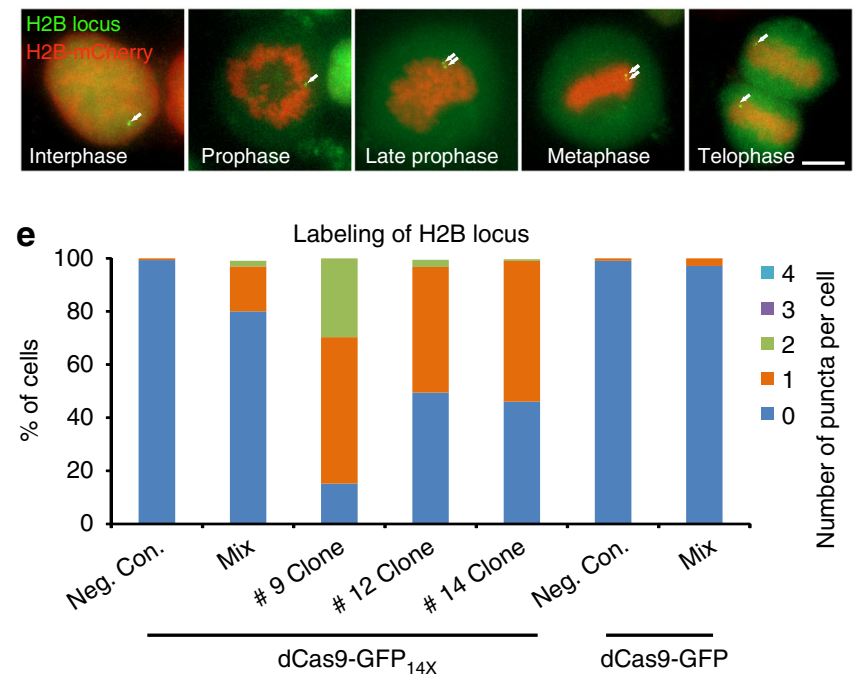

g

Labeling of LMNA locus

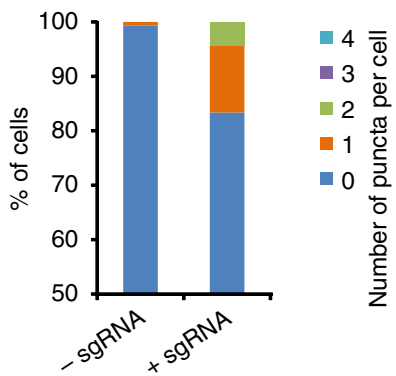

Fig. 1 Development of CRISPR-Tag to label non-repetitive genes. a Schematic of CRISPR-Tag design as a DNA tagging system. b Co-expression of four sgRNAs in one vector. sgTS1, sgTS2, sgTS3, and sgTS4 were built individually and then sub-cloned into a single vector. c mCherry-CRISPR-Tag_v1 was inserted into $\mathrm{C}$-terminus of human $\mathrm{H} 2 \mathrm{~B}$ gene as highlighted by yellow. Representative images denoted simultaneous visualization of $\mathrm{H} 2 \mathrm{~B}-\mathrm{mCherry}$ and $\mathrm{H} 2 \mathrm{~B}$ loci by using CRISPR-Tag. H2B loci were labeled by dCas9-GFP $14 x$ with four sgRNAs (sgTS1-sgTS4). $\mathbf{d}$ H2B-mCherry and H2B loci were visualized at different stages of cell cycles. e Quantifications of H2B locus labeling efficiency, $n \geq 84$ cells. $\mathbf{f}$ Representative images to show simultaneous visualization of mCherry-LMNA and LMNA loci by utilizing the CRISPR-Tag system. $\mathbf{g}$ Quantifications of LMNA locus labeling efficiency, $n \geq 150$ cells. All images in c, $\mathbf{d}$, and $\mathbf{f}$ are maximum intensity projections from z stacks. Scale bars: $5 \mu \mathrm{m}$

(sgTS1-sgTS4) (Fig. 1b, c). Histone H2B, as one of the five main histone proteins, is involved in the formation of chromatin structure in eukaryotic cells ${ }^{23}$. H2B-mCherry allowed imaging of both interphase chromatin and mitotic chromosomes and revealed various chromatin states. In the meanwhile, we observed that $\mathrm{H} 2 \mathrm{~B}$ locus underwent replication and separation into two daughter cells at the telophase stage (Fig. 1d; Supplementary Movie 1). We observed that $\sim 17 \%$ of $\mathrm{H} 2 \mathrm{~B}$-mCherry ${ }^{+}$mix pool cells showed a single, clearly GFP-tagged spot, indicating only one of the alleles was successfully modified by CRISPR editing. $2 \%$ of cells have two spots, which might be due to DNA replication during the cell cycle or modifications of both alleles. The other $80 \%$ of cells did not display clear fluorescent spots, which might be attributed to sub-optimal expression of dCas9-GFP $11_{14 \mathrm{x}}$, GFP1-10, and/or sgRNA in this mixed pool. We isolated three single cell clones which showed specific labeling (Clones 9, 12, 
and 14). All three had high labeling efficiency, 85\%, 51\%, and $54 \%$, respectively (Fig. 1e; Supplementary Fig. 7). Nearly $100 \%$ of labeled cells in Clone 12 and 14 only contained a single spot, while about $30 \%$ of labeled cells in Clone 9 showed two spots. The difference is likely due to the number of modified alleles (Supplementary Fig. 6e). Taken together, we suggest that clonal isolation is ideal for increasing labeling efficiency, but is not necessary.

Next, we asked if signal amplification using tandem split GFP is a must for CRISPR-Tag system. To address this question, we carried out CRISPR-Tag knock-in to label H2B locus in dCas9EGFP cells. No clearly visible spots were observed, suggesting that signal amplification using dCas9-GFP ${ }_{14 \mathrm{X}}$ is critical in this DNA tagging system (Fig. 1e). Similarly, we achieved simultaneous imaging of protein and gene positioning for both human nuclear membrane protein LMNA, and heat-shock response protein HSPA8 in living cells by CRISPR-Tag system (Fig. 1f, g; Supplementary Figs. 5d, f and 8).

CRISPR-Tag in introns minimally affects gene expression. Specifically when tagging protein-coding genes, it is possible that certain $3^{\prime}$ UTR sequences may affect the expression level. Indeed, comparing to inserting mCherry alone, inserting mCherry together with CRISPR-Tag caused a decrease in protein expression level for H2B, LMNA, and HSPA8 in HeLa cells, but not H2B in 293 T cells (Fig. 2; Supplementary Fig. 9). To minimalize this effect, we engineered the mCherry coding sequence to add in an intron while maintaining its expression level (Supplementary Fig. 10a, b). We have also assembled a second version of CRISPRTag, termed CRISPR-Tag_v2, which can be recognized by sgTS5, sgTS6, and sgTS7. We designed two different lengths of CRISPR-
Tag_v2, containing 4 and 6 repeats, respectively (Fig. 2a, b). When inserting in the mCherry intron, CRISPR-Tag_v2 had no effect on H2B-mCherry expression, whereas our earlier design CRISPR-Tag_v1 caused a decrease in mCherry expression, possibly due to its interference with splicing (Supplementary Figs. 10c, d).

Then, we validated CRISPR-Tag for the labeling and imaging of $\mathrm{H} 2 \mathrm{~B}$ and LMNA genes. With this strategy, both $\mathrm{H} 2 \mathrm{~B}$ and LMNA genes could be efficiently labeled using three sgRNAs while not affecting their expression levels (Fig. 2c, d; Supplementary Fig. 11). To further confirm whether CRISPR-Tag insertions disrupt gene transcription, we compared the transcription level of the fluorescent fusion of $\mathrm{H} 2 \mathrm{~B}$ and LMNA with or without CRISPR-Tag insertions. Relative RNA expression was normalized against endogenous genes (Supplementary Fig. 12). We did not observe significant differences between the control and samples, indicating that our strategy generates no obvious disruption to transcription. It is worth noting that quantitative PCR results indicated that CRISPR-Tag in UTR region has little effect on transcription, which is probably due to the limit of qPCR detection. FACS analysis directly quantifies protein expression level of CRISR-Tag knock-in allele, while qPCR measures transcription of all alleles in the cell.

Because dCas9-GFP $14 \mathrm{X}$ would be much larger than a single GFP tag, we tested whether the labeling of protein-coding genes by dCas9-GFP ${ }_{14 \mathrm{x}}$ could affect protein expression. We compared the expression of H2B-mCherry in cells with or without CRISPRpositive signal and found no significant difference (Supplementary Fig. 13). Together, these results suggest that the CRISPR-Tag system does not obviously perturb gene expression. a

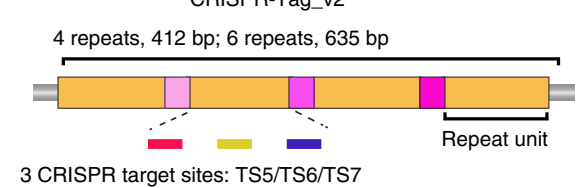

C
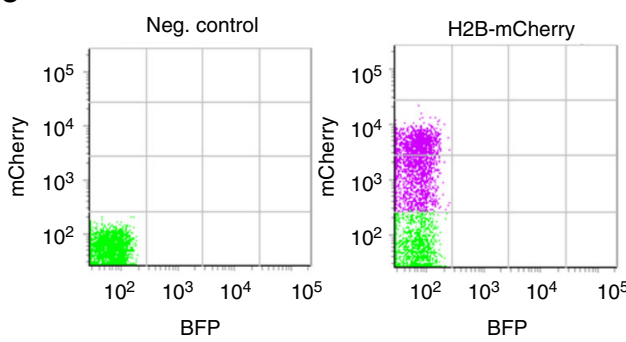

d
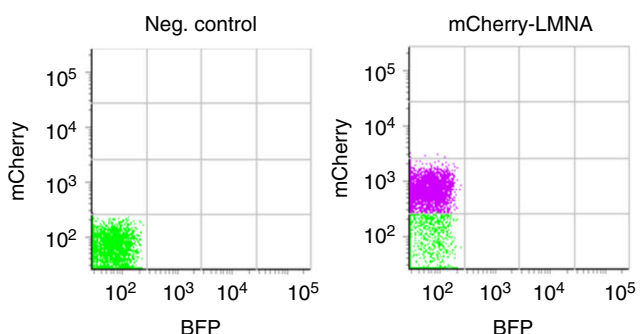

b
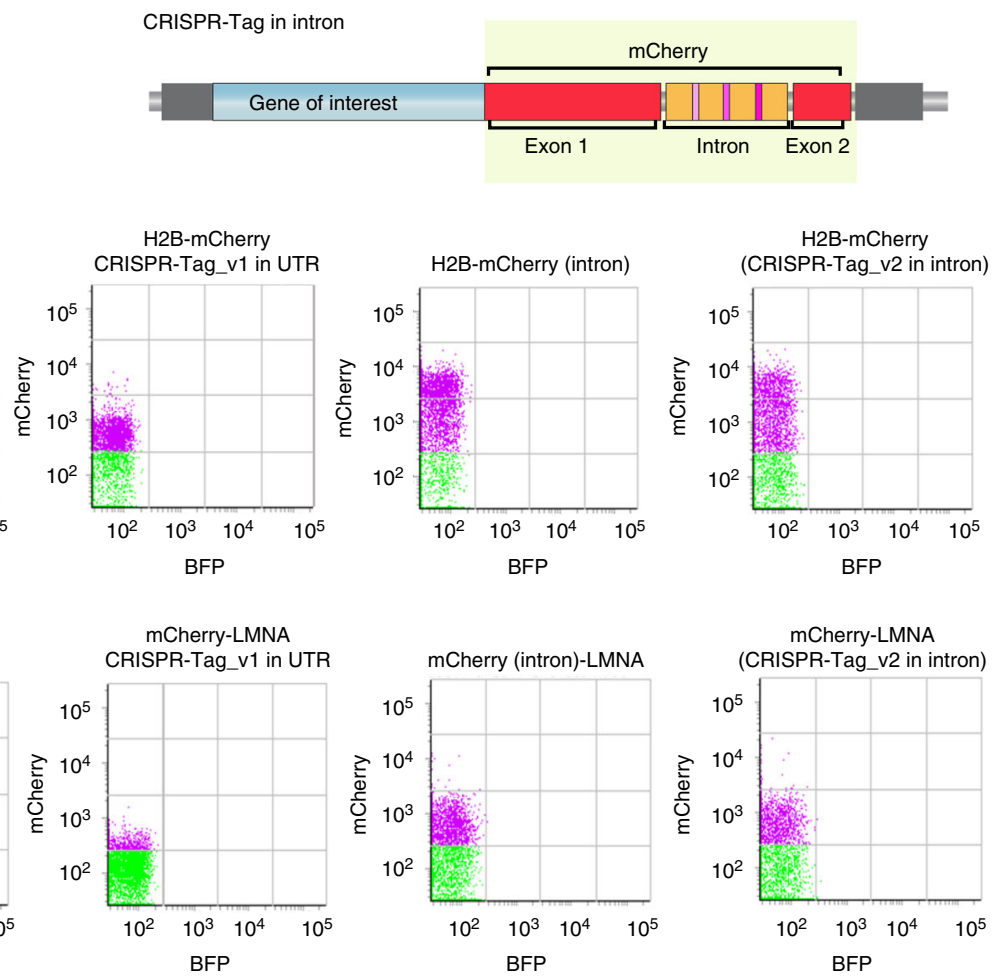

Fig. 2 The protein expression remains normal when hiding CRISPR-Tag in intron. a Schematic of CRISPR-Tag_v2 design which contains four repeats. Each repeat harbors three CRISPR-targeting sites including TS5, TS6, and TS7. b Schematic of CRISPR-Tag positioning in the intron of mCherry. The fragment including mCherry and CRISPR-Tag, indicated by yellow, was integrated to the N-terminus or C-terminus of a target gene. c, $\mathbf{d}$ FACS analysis was performed to characterize H2B-mCherry (c) or mCherry-LMNA (d) expression in HeLa cells. Each dot represents single cells. BFP serves as an irrelevant channel. The distribution of dots along the $y$-axis indicates mCherry signal intensity 
CRISPR-Tag allows long-term tracking of DNA and protein. There is no doubt that the longer the CRISPR-Tag is, the higher SNR can be achieved. Using CRISPR-Tag_v2 $6 x$ (six repeats, 635 bp) to label H2B locus, $19 \%$ of mix pool cells had clearly spots, while this number reduced to $10 \%$ using CRISPR-Tag_v $2_{4 \mathrm{x}}$ (four repeats, $412 \mathrm{bp}$ ). Similar results were observed for labeling LMNA locus (Fig. $3 \mathrm{a}-\mathrm{c}$ ). Furthermore, CRISPR-Tag_v2 $6 \mathrm{x}$ achieves relatively higher SNR, especially when labeling H2B locus (Fig. 3d). Obviously, the detection efficiency and quality can be further enhanced by using confocal microscopy (Fig. 3e). We performed short-term uninterrupted imaging to capture the dynamics of replicated LMNA loci and found that the CRISPR signal is stable (Supplementary Movie 2). Long-term live cell imaging was also carried out to track the dynamics of LMNA locus and mCherryLMNA throughout the cell cycle (Supplementary Movie 3). Spinning-disk confocal imaging captured the entire process of breaking-down and reconstruction of mCherry-LMNA-labeled nuclear membrane, and also the duplication and separation of LMNA locus during mitosis. These results further demonstrate the applicability of our approach to capture long-term dynamics of DNA and protein of a specific gene throughout the cell cycle.

Assess CRISPR-Tag designs for effective DNA labeling. We next performed a series of optimizations and quantifications in order to reduce the size of the CRISPR-Tag while maintaining its labeling efficiency. First, we compared signal-to-noise ratio by using different numbers of sgRNAs to label CRISPR-Tag_v1, which is $803 \mathrm{bp}$ long containing six repeats. Each repeat can be recognized by up to four sgRNAs (sgTS1-sgTS4). We observed that transfection of one single sgRNA, such as sgTS1, sgTS2, and sgTS3, resulted in sufficient visualization of CRISPR-Tag with SNR above 15. The SNR increased to $23.3 \pm 8.7$ and $28.6 \pm 10.1$ (mean \pm SEM) when using two and four sgRNAs, respectively (Fig. 4a, b). Collectively, one sgRNA to target six sites is sufficient for labeling a specific locus. We next sought to further reduce the size of CRISPR-Tag by assembling fewer repeats, including 5, 3, 2, and 1 repeats. We found that the size of CRISPR-Tag can be as small as 251 bp harboring four CRISPR-targeting sites (Fig. 4c). However, it is important to note that shorter CRISPR-Tag resulted in lower SNR (Fig. 4d). Therefore, our results suggest using relatively longer CRISPR-Tag to achieve effective labeling. It is also important to optimize the spacing of two adjacent targeting sites in the CRISPR-Tag because steric hindrance of neighboring dCas 9 protein-binding sites is a concern. We varied the spacing length and quantified SNR. The results indicated that $52 \mathrm{bp}$ spacing linker separating the two neighboring targeting sites achieved the best result (Fig. 4e, f). Together, our studies provide some critical guidelines for CRISPR-Tag design. The smallest CRISPR-Tag is substantially shorter than the LacO/TetO array (usually $\sim 10$ and $\sim 4 \mathrm{~kb}$, respectively) ${ }^{14}$, and the ParB/INT $(\sim 1 \mathrm{~kb})$ system $^{24}$.

\section{Discussion}

By assembling C. elegans genomic sequences which can be effectively targeted by the CRISPR-Cas9 system, we created CRISPR-Tag as a DNA tagging tool in living human cells, enabling live-cell labeling of non-repetitive genes at the level of single cells and single loci. Although these tags are as small as 251 bp, longer ones such as CRISPR-Tag_v2 (635 bp) do lead to higher SNR, which would be the optimal choice for most applications. Our CRISPR-Tags are consisted of no more than six repeats (24 CRISPR target sites). For comparison, a recent paper demonstrated an optimized $\mathrm{TetO}$ repeat for labeling endogenous loci while emphasizing that 96 repeats of TetO was required for long-term imaging ${ }^{25}$. The small size and low-repetitiveness of our
CRISPR Tags are advantageous because they are less likely to perturb the chromatin structure or affect transcription and replication. It is possible that the repeat number of TetO can also be reduced using our signal amplification approach, although whether the 14x GFP labeling of TetR will affect its affinity to TetO needs to be tested.

All the CRISPR-Tags we created in this study are smaller than $850 \mathrm{bp}$ and as small as $251 \mathrm{bp}$. Thus, CRISPR-Tag represents the smallest DNA tags among all the available tags. As a proof of concept, we developed a DNA tagging strategy, engineering CRISPR-Tag in the intron region of a fluorescent protein. Although this approach requires modification of endogenous loci, it has no significant effect on both gene transcription and protein expression, at least for the labeling of human H2B and LMNA. Systematic examination of even more protein-coding genes could further confirm this characteristic. Most importantly, our strategy enables simultaneously imaging of both DNA and protein expression of a specific gene. Thus, this imaging platform will provide a tool to uncover how gene positioning and movement play a role in gene regulation during development and disease progression.

An alternative ParB/INT system, also called "ANCHOR DNA labeling system (ANCHOR3)", is based on insertion of a nonrepetitive DNA sequence $(\mathrm{ANCH}, \sim 1 \mathrm{~kb})$ to which $\mathrm{OR}$ (bacterial partition protein or ParB) bind site-specifically ${ }^{24}$. Oligomerization of OR-FP fusion proteins lead to accumulation of fluorescent proteins, which non-specifically and dynamically associate with adjacent DNA. Although AHCHOR DNA tag is short and nonrepetitive, whether the spreading of OR proteins to adjacent genomic region has any effects on the target region need to be carefully addressed. In addition, it is unknown whether AHCHOR DNA tag can work well for labeling endogenous genes in human cells. What remains to be done is the direct comparing of all the different DNA tags.

Our results indicated that sgRNAs successfully targeting $C$. elegans genome also work effectively in human cells. In theory, CRISPR-Tag approach can be applied broadly for any other species, such as using Human CRISPR-Tag for C. elegans genomic loci labeling. Furthermore, a same set of CRISPR-Tag and sgRNAs is applicable for tagging any genomic loci. This is an advantage over previous CRISPR imaging technique which requires at least 26 sgRNAs $^{6}$. The labeling efficiency depends on how many of the sgRNAs are functional and how many are delivered into the cells. The number of sgRNAs required for nonrepetitive DNA labeling might be reduced using super-resolution microscopy ${ }^{9,10}$. However, off-target or unspecific labeling needs to be carefully assessed if fewer sgRNAs are used. Combining dCas9-GFP ${ }_{14 \mathrm{X}}$ and CRISPR-Tag, DNA labeling was achieved by targeting 4-24 sites in our system. Obviously, the more target sites, the stronger signal would be detected.

Compared to previous CRISPR imaging technique, the disadvantage of CRISPR-Tag system is the additional knock-in step. Due to the low knock-in efficiency, we observed low frequency of clones with biallelic knock-in. This can be optimized by using novel CRISPR-mediated knock-in strategy, such as use of different types of donor DNA or different ways of repairing mechanisms ${ }^{26,27}$. Nevertheless, CRISPR-Tag provides DNA labeling at the level of single locus, which can be useful for some cases, such as labeling imprinted genes. One limit of CRISPR-Tag strategy is its use for tagging regulatory elements, such as enhancers, which is a general problem for all DNA tags. TetO repeats were integrated $16 \mathrm{~kb}$ far away from target genes ${ }^{25}$. ANCH tag was inserted adjacent to the promoter of the transgene $^{24}$. To our knowledge, no DNA tags have been applied to label regulatory elements. Previous CRISPR imaging technique might be the best way to label regulatory elements. Important to 
a
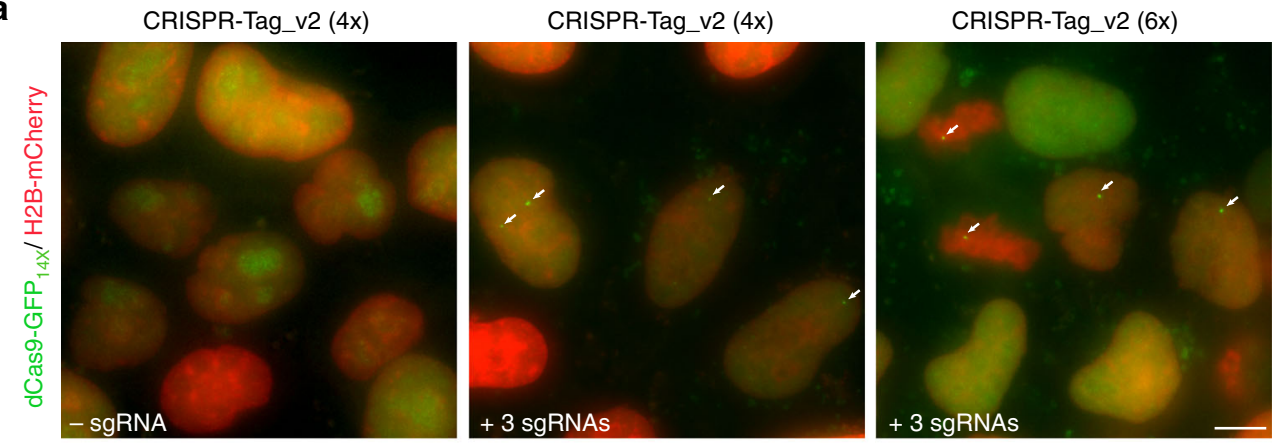

b
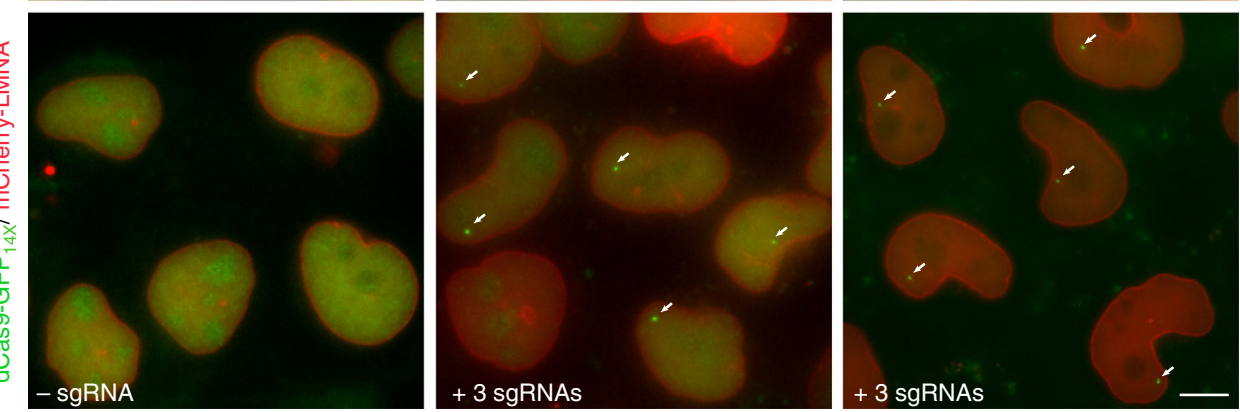

C
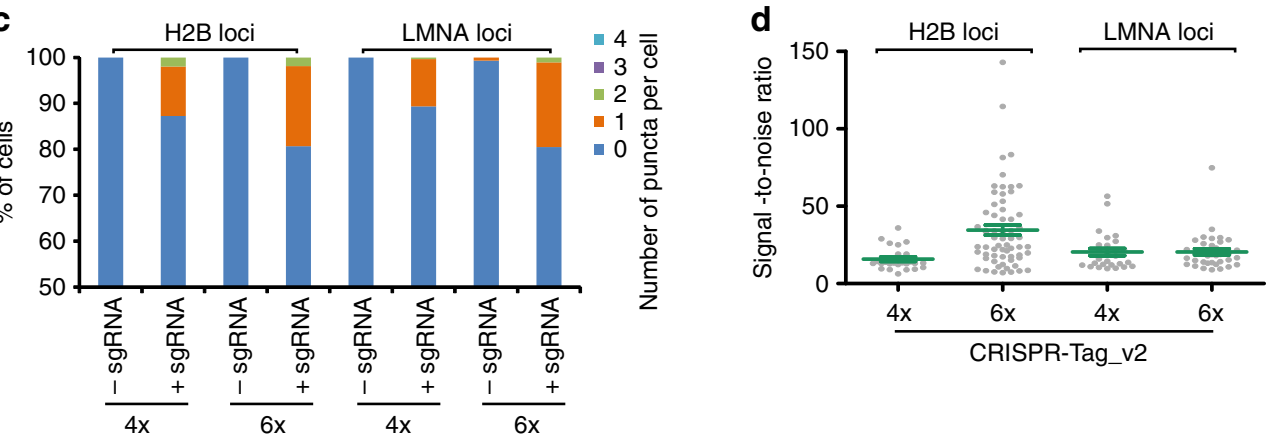

e
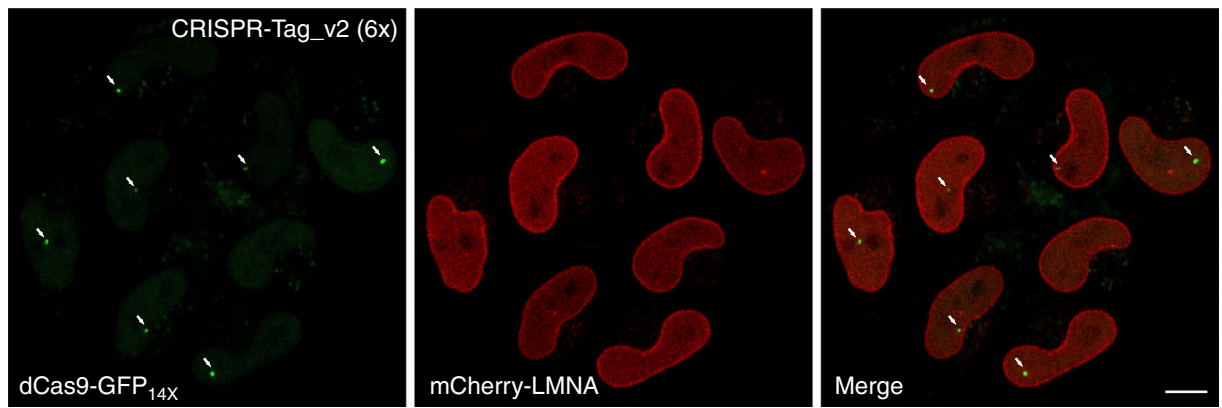

Fig. 3 CRISPR-Tag in intron enables simultaneous visualization of DNA and protein of endogenous genes. a Visualization of H2B locus using CRISPRTag_v2 (containing four or six repeat units), which was inserted into the intron of mCherry. H2B-mCherry was imaged and H2B loci were labeled by dCas9GFP $_{14 X}$ with three sgRNAs (sgTS5-sgTS7). b Visualization of LMNA locus using CRISPR-Tag_v2 with three sgRNAs (sgTS5-sgTS7). c Labeling efficiency of $\mathrm{H} 2 \mathrm{~B}$ and LMNA loci shown in $\mathbf{a}$ and $\mathbf{b}$, respectively, was determined by counting the number of spots in each cell, $n \geq 191$ cells. $\mathbf{d}$ Labeling efficiency of $\mathrm{H} 2 \mathrm{~B}$ and LMNA loci was determined by quantifying signal-to-noise ratio, $n \geq 25$ cells. Green line denotes means \pm SEM. e Confocal images of mCherry-LMNA and LMNA loci labeled by dCas9-GFP $14 x$ and CRISPR-Tag_V2 (6x). All images in $\mathbf{a}, \mathbf{b}$, and $\mathbf{e}$ are maximum intensity projections from z stacks. Scale bars: 10 $\mu \mathrm{m}$

note, an ideal DNA tagging system should not interfere with the function of the target loci. The binding of Cas9 to the target DNA begins with DNA unwinding to form an RNA-DNA hybrid, which may affect the localization of histones and other DNAbinding proteins ${ }^{28,29}$. However, Cas9 binding to DNA is reversible $^{30}$, thus Cas 9 could be replaced by other players. Our previous studies suggested dCas9 binding to telomeres did not apparently affect telomere integrity and their movement dynamics ${ }^{6}$. Nevertheless, the effects of dCas9 binding to the target loci remain to be fully addressed.

Orthologous CRISPR systems, with their different PAM requirements, provide a powerful platform for achieving multicolor labeling of genomic loci. Cas9 orthologs, SpCas9 and SaCa9, from Streptococcus pyogenes and Staphylococcus aureus, respectively, have been applied for labeling three different elements in one cell ${ }^{31}$. Alternatively, sgRNA scaffolds could be adapted to recruit different 
a

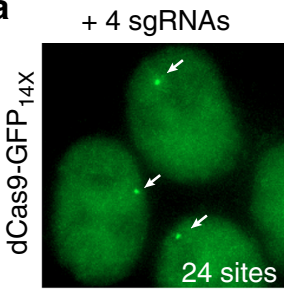

$+\operatorname{sgTS} 2$

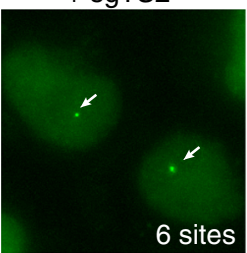

C
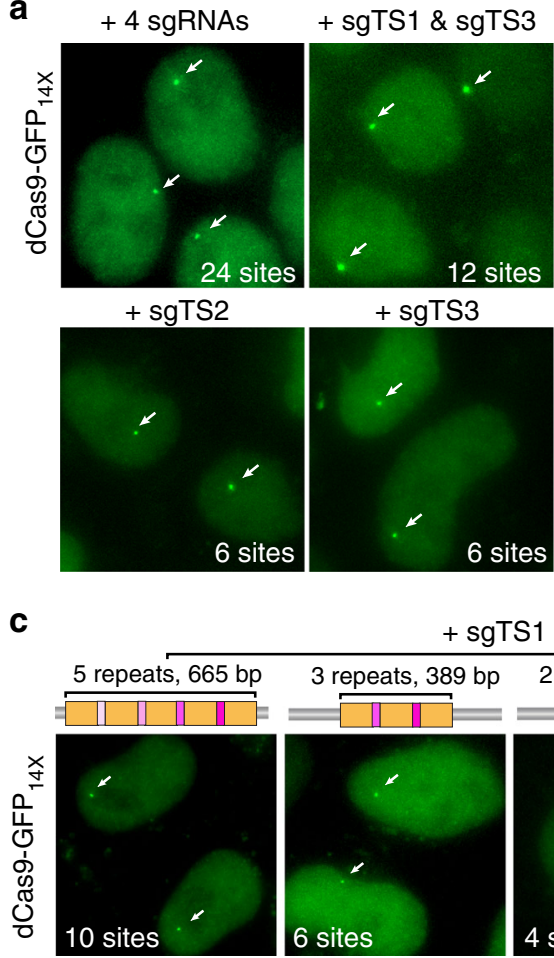

$+\operatorname{sgTS3}$

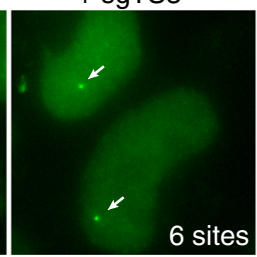

+ sgTS1 \& sgTS3
+ sgTS1

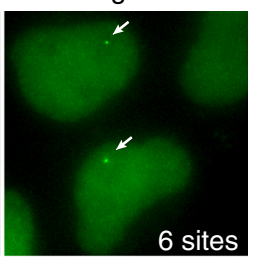

+ sgTS4

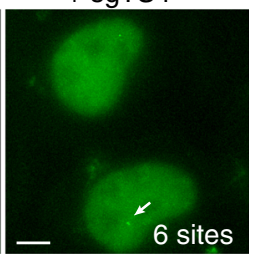

b

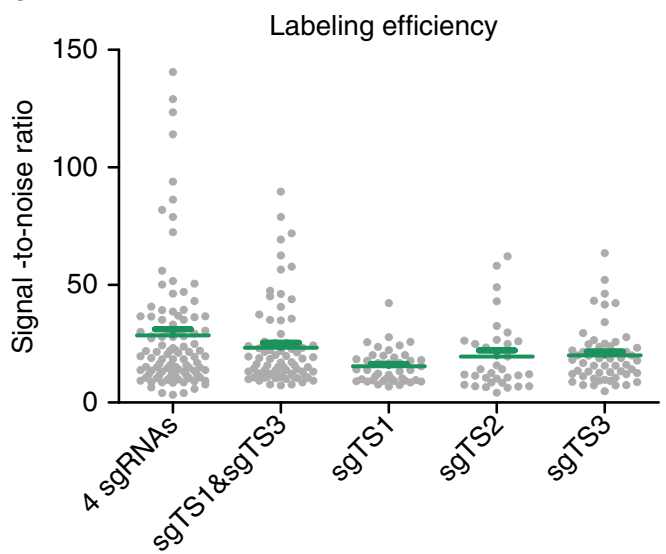

d

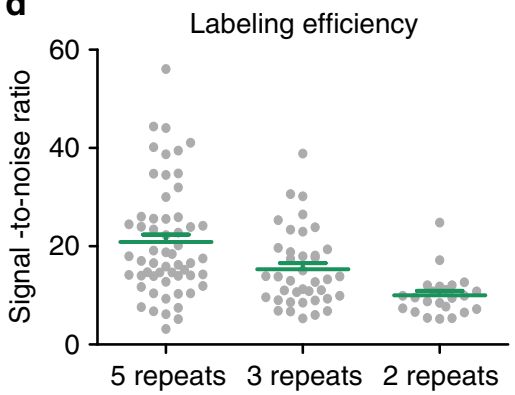

$\mathbf{f}$

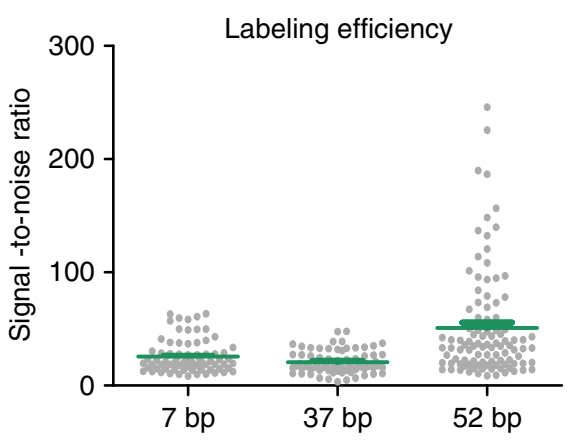

Fig. 4 Assess CRISPR-Tag designs for effective DNA labeling. a Minimal number of sgRNAs to label H2B loci with CRISPR-Tag_v1. The use of four sgRNAs allows 24 binding sites of dCas9-GFP $14 x$ and one sgRNA facilitates six CRISPR-targeting sites. Representative images were shown for each case. Detected $\mathrm{H} 2 \mathrm{~B}$ loci are indicated by arrows. b Comparison of labeling efficiencies of using different sgRNAs, $n \geq 33$ cells. Error bars represent \pm SEM. c Minimal number of CRISPR-targeting sites to label H2B loci. CRISPR-Tag containing different copies of repeat units were generated and then labeled by expressing both sgTS1 and sgTS3, respectively. d Labeling efficiency was defined by quantifying signal to noise ratio, $n \geq 23$ cells. Green line reports means \pm SEM. e Optimal spacing length between two neighboring targeting sites. Spacing length was defined as the number of nucleotides from NGG to next protospacer sequence as indicated in the diagram. $\mathbf{f}$ Labeling efficiency was defined by quantifying signal to noise ratio, $n \geq 72$ cells. Green line reports means \pm SEM. All images in $\mathbf{a}, \mathbf{c}$, and $\mathbf{e}$ are maximum intensity projections from z stacks. Scale bars: $5 \mu \mathrm{m}$

fluorescent proteins using MS2/PP7 motifs $^{32,33}$. Thus, a wide range of orthogonal CRISPR-Tags with different CRISPR-targeting sequences can be assembled to achieve multi-color labeling. Considering that signal amplification using tandem split GFP greatly enhance the labeling efficiency, it would be useful to adapt Sun$\mathrm{Tag}^{34}$ system and split mCherry ${ }^{35}$ for generating more colors. Taken together, CRISPR-Tag opens up possibilities for both genomic tool developments and biological applications.

\section{Methods}

Cell culture. HeLa and HEK293T cells (obtained from UCSF Cell Culture Facility) were maintained in Dulbecco's modified Eagle medium (DMEM) with high glucose
(Gibco) in 10\% FBS (Clontech) and 1\% penicillin/streptomycin (Gibco). All cells were grown at $37{ }^{\circ} \mathrm{C}$ and $5 \% \mathrm{CO}_{2}$ in a humidified incubator.

Construction of dCas 9 and sgRNA. The nuclease-deficient $S$. pyogenes Cas 9 (dCas9) was used for all the CRISPR imaging experiments in this study. The construction of dSpCas9-EGFP has been previously described ${ }^{6}$. To build dCas9GFP $11_{14 x}$, we first synthesized two fragments of gBlocks, each containing seven copies of the coding sequence of GFP11 tag. Then we modified the SunTag vector (pHR-dSV40-NLS-dCas9-24xGCN4_v4-NLS-P2A-BFP-dWPRE, addgene \#60910) to replace $24 \mathrm{xGCN} 4$ with GFP11 $14 \mathrm{x}$. The DNA sequence encodes dCas9-GFP11 $14 \mathrm{X}$ was provided in supplementary table 3. To express GFP1-10 in the nucleus, we fused GFP1-10 with one copy of SV40 NLS and cloned the fusion protein into a lentiviral vector containing a strong promoter $P_{\mathrm{SFF}}$. The DNA sequence of GFP110 was provided in supplementary table 4 . sgRNAs to label MUC4 and 5S rDNA genes (Supplementary Figs. 2 and 3 ) were cloned into a lentiviral vector (AddGene 
\#51024). Other sgRNAs in this study, including sgTS1-sgTS7, were built by modifying the CRISPRainbow vector (addgene \#75398). For co-expression of multiple sgRNAs $(>2)$, the Golden Gate Cloning method was used to assemble multiple sgRNAs into CRISPRainbow-donor vector (AddGene \#75398) ${ }^{32}$. All the sgRNA-targeting sequences were listed in supplementary table 5.

Assembly of CRISPR-Tag. To assemble the CRISPR-Tag, a gBlock containing a series of C. elegans genomic sequences (GNNNNNNNNNNNNNNNNNNNNGG) that can be recognized by CRISPR-Cas9 system was synthesized by Integrated DNA Technologies (IDT). The spacing of two neighboring CRISPR-targeting sits varies according to the design. To assemble a CRISPR-Tag with a desired number of repeats, the repeat unit containing 3 or 4 CRISPR-targeting sites was amplified by PCR reactions using the Phusion High-Fidelity DNA polymerase (New England Biolabs). The Golden Gate Cloning method was then performed to assemble CRISPR-Tags that consist of different desired numbers of repeats (diagram in supplementary Fig. 1). Important to note, there is a unique sequence arranged between two adjacent repeats in the CRISPR-Tag, which facilitates easy validation of CRISPR knock-in by PCR reactions. The sequences of CRISPR-Tag_vl and CRISPR-Tag_v2 were provided in supplementary table 6.

Re-engineering mCherry for carrying the CRISPR-Tag. The fourth intron of human HSPA5 gene was inserted into mCherry-coding sequence right after the three nucleotides that code for the 197th amino acid of mCherry protein. A restriction site BstXI was artificially embedded in the intron region for the ease of molecular cloning. CRISPR-Tag was then cloned into the BstXI site by In-Fusion HD Cloning Kit (Clontech). The sequences of mCherry fusions with intron and CRISPR-Tag were provided in supplementary table 7 .

Construction of donor plasmids. All donor plasmids used in this study were generated with NEBuilder HiFi DNA Assembly Cloning Kit (New England Biolabs). For example, to construct the donor plasmid for inserting mCherry and CRISPR tag to the C-terminus of $\mathrm{H} 2 \mathrm{~B}$, the left and right homology arm (HA) were amplified from the genomic DNA of HeLa cells, with the stop codon being removed. Other two fragments, mCherry and CRISPR tag were amplified. Then the four fragments, including left HA, mCherry, CRISPR-Tag, and right HA were assembled into a same vector (diagram in Supplementary Fig. 1). To generate donor plasmids harboring $\mathrm{sgH} 2 \mathrm{~B}$ recognition sites, termed double-cut donor plasmid, the sgH2B-targeting sequence together with the PAM sequence (GCGAGCGCCAGGTCCCGGCAGGG) was included in the forward primer of left $\mathrm{HA}$ and the reverse primer of $\overline{\text { right }} \mathrm{HA}$. Therefore, sgH2B-targeting sequence was tagged to the regions flanking the upstream and downstream HA.

Lentiviral production. HEK293T cells were seeded into six-well plates $24 \mathrm{~h}$ prior to transfection. $110 \mathrm{ng}$ of pMD2.G plasmid, $890 \mathrm{ng}$ of pCMV-dR8.91, and $1000 \mathrm{ng}$ of the lentiviral vector (dCas9-EGFP, dCas9-GFP11 $14 \mathrm{x}, \mathrm{GFP} 1-10$ or sgRNA) were cotransfected into HEK293T in each well using FuGENE (Promega) following the manufacture's recommended protocol. Virus was harvested $48 \mathrm{~h}$ after transfection.

Transfection, infection, and generation of stable cell lines. HeLa cells were infected with dCas9-EGFP and Tet-on 3G lentiviruses, and then clonal cell lines were isolated to express dCas9-EGFP at a suitable level following our previous protocol $^{36}$. We diluted the cells and distributed $0-1$ cell into each well of 96-well plate. After 10-14 days, selected colonies were tested for DNA labeling. The basal expression level of dCas9-EGFP without doxycycline induction is ideal to achieve high signal-to-noise ratio. To generate HeLa cells stably expressing dCas9-GFP ${ }_{14 \mathrm{x}}$, we infected HeLa cells with dCas9-GFP $14 \mathrm{x}$ and GFP1-10 lentivirues. A clonal cell line which achieved the best signal-to-noise ratio was selected for CRISPR imaging. The non-repetitive region of MUC4 gene was labeled by infecting dCas9-GFP expressing cells with a mixed lentiviral cocktail containing 36 sgRNAs. $3 \mu \mathrm{g}$ of the purified plasmid cocktail (5-6 sgRNAs per cocktail), $0.33 \mu \mathrm{g}$ of pMD2.G and $2.66 \mu \mathrm{g}$ of pCMV-dR8.91 were cotransfected into HEK293T cells seeded in T25 flask for creating a mixed lentiviral cocktail. 48-h postransfection, seven lentiviral cocktails for these 36 sgRNAs were generated and mixed (with equal amounts of each cocktail). dCas9-GFP-expressing cells were then infected with lentiviral mixtures in eight-well of chambered coverglass. To enhance the infection of each lentivirus, a higher dosage of virus (2:3 dilution) in the presence of polybrene $(5 \mu \mathrm{g} / \mathrm{mL})$ was used for non-repetitive DNA labeling. To label repetitive genomic elements or specific protein-coding genes, dCas9-FP stable cell line without/with successful knock-in of CRISPR-Tag was transfected with sgRNAs using FuGENE (Promega) following the manufacturer's recommended protocol. For all the CRISPR imaging experiments, HeLa cells were plated into eight-well chambered coverglass (Lab-Tek II). $400 \mathrm{ng}$ of total sgRNA plasmid DNA were transfected for each individual well.

sgRNA in vitro transcription. sgRNAs for CRISPR-mediated knock-in was transcribed in vitro following the published protocol ${ }^{37}$. The following sequence was used as the DNA template to transcribe sgRNAs in vitro: $5^{\prime}$-TAA TAC GAC TCA CTA TAG GNN NNN NNN NNN NNN NNN NNG TTTAAG AGC TAT GCT GGA AAC AGC ATA GCA AGT TTA AAT AAG GCT AGT CCG TTA TCA
ACT TGA AAA AGT GGC ACC GAG TCG GTG CTT TTT TT-3' containing a T7 promoter (TAATACGACTCACTATAG), a gene specific $\sim 20$-nt protospacer sequence starting with a $\mathrm{G}$ for optimal T7 transcription (GNNNNNNNNNNNN NNNNNNN), and a common sgRNA scaffold region. The DNA template was generated by overlapping PCR using a set of four primers: three primers common to all reactions (forward primer T25: 5'-TAA TAC GAC TCA CTA TAG-3'; reverse primer BS7: 5'-AAA AAA AGC ACC GAC TCG GTG C-3' and reverse primer ML611: 5'-AAA AAA AGC ACC GAC TCG GTG CCA CTT TTT CAA GTT GAT AAC GGA CTA GCC TTA TTT AAA CTT GCT ATG CTG TTT CCA GCA TAG CTC TTA AAC- ${ }^{\prime}$ ) and one gene-specific primer (forward primer $5^{\prime}$ TAA TAC GAC TCA CTA TAG GNN NNN NNN NNN NNN NNN NNG TTT AAG AGC TAT GCT GGA A-3'). For each template, a $100 \mu \mathrm{L}$ PCR was set using iProof High-Fidelity Master Mix (Bio-Rad) reagents. The PCR product was then purified using DNA Clean and Concentrator- 5 columns (Zymo primer (forward primer 5'-TAA TAC GAC TCA CTA TAG GNN NNN NNN NNN NNN NNN NNG TTT AAG AGC TAT GCT GGA A-3'). For each template, a $100 \mu \mathrm{L}$ PCR was set using iProof High-Fidelity Master Mix (Bio-Rad) reagents. The PCR product was then purified using DNA Clean and Concentrator-5 columns (Zymo Research) following the manufacturer's instructions. Next, a $100 \mu \mathrm{L}$ in vitro transcription reaction was carried out using HiScribe T7 High Yield RNA Synthesis Kit (New England Biolabs). The sgRNA product was then purified on RNA Clean and Concentrator-5 columns (Zymo Research) and eluted in $15 \mu \mathrm{L}$ of RNase-free RNA buffer $\left(10 \mathrm{mM}\right.$ Tris $\mathrm{pH} 7.0$ in DEPC-treated $\left.\mathrm{H}_{2} \mathrm{O}\right)$. sgRNA quality was examined by running 3 pg of the purified sgRNA on a $10 \%$ polyacrylamide gel containing $7 \mathrm{M}$ urea (Novex TBE-urea gels, ThermoFisher Scientific).

CRISPR-mediated knock-in. RNP assembly and electroporation were performed to carry out CRISPR knock-in experiments. Cas9/sgRNA RNP complexes were prepared following methods reported by Lin et al..$^{21}$. Cas9 protein (pMJ915 construct, containing two nuclear localization sequences) was expressed in E. coli and purified by the University of California Berkeley Macrolab following protocols described by Jinek et al. ${ }^{3}$. The HeLa dCas9-GFP ${ }_{14 \mathrm{x}}$ cells were treated with $200 \mathrm{ng} /$ $\mathrm{mL}$ nocodazole (Sigma) for $15 \mathrm{~h}$ prior to electroporation to increase HDR efficiency. RNP complexes were assembled with 100 pmol Cas 9 protein and $130 \mathrm{pmol}$ sgRNA just before electroporation and combined with 400 ng donor plasmid DNA in a final volume of $10 \mu \mathrm{L}$. First, 130 pmol-purified sgRNA was diluted in Cas9 buffer (final concentrations: $150 \mathrm{mM} \mathrm{KCl}, 20 \mathrm{mM}$ Tris pH 7.5, $1 \mathrm{mM} \mathrm{TCEP}-\mathrm{HCl}$, $1 \mathrm{mM} \mathrm{MgCl} 2,10 \% \mathrm{vol} / \mathrm{vol}$ glycerol $)$ and incubated at $70^{\circ} \mathrm{C}$ for $5 \mathrm{~min}$. A total of 2.5 $\mu \mathrm{L}$ of Cas9 protein $(40 \mu \mathrm{M}$ stock in Cas 9 buffer, i.e., $100 \mathrm{pmol})$ was then added and RNP assembly was carried out at $37^{\circ} \mathrm{C}$ for $10 \mathrm{~min}$. Finally, donor plasmid DNA was added to this RNP solution. Electroporation was performed in a Amaxa 96well shuttle Nuleofector device (Lonza) using SF-cell line reagents (Lonza) following the manufacturer's instructions. Nocodazole-treated HeLa cells were washed with PBS and resuspended to $10^{4}$ cells per microliter in SF solution immediately before electroporation. For each sample, $20 \mu \mathrm{L}$ of cells (i.e., $2 \times 10^{5}$ cells) was added to the $10 \mu \mathrm{L} \mathrm{RNP} /$ template mixture. Cells were immediately electroporated using the CM130 program and transferred to $1 \mathrm{~mL}$ pre-warmed culture medium in a 24-well plate. FACS selection of knock-in positive cells were carried out 3 days after electroporation.

Flow cytometry. Three days following Cas9/sgRNA/donor electroporation, cells were analyzed by flow cytometry on aLSR II instrument (BD Biosciences) and sorted on a FACSAria II (BD Biosciences). Cells were first gated for the intact cell population using forward scatter versus side scatter plots and then gated for single cells based on forward scatter $\mathrm{W}$ versus forward scatter $\mathrm{H}$. mCherry-positive cells were sorted out for further validation of CRISPR knock-in.

Quantitative RT-PCR. Cells were harvested using trypsin (Hyclone), and total RNA was isolated using FastPure Cell/Tissue Total RNA Isolation Kit (Vazyme), according to manufacturer's instructions. RNA was converted to cDNA using oligo-dT primers (HiScript II Q RT SuperMix for qPCR, Vazyme). Quantitative PCR reactions were prepared using ChamQ Universal SYBR qPCR Master mix (Vazyme). Reactions were run on the QuantStudio 5 Real-Time PCR system (Thermo Fisher). All reactions were performed in triplicate. RNA abundance was normalized to an endogenous reference gene UBC and calculated as delta-delta threshold cycle $(\Delta \Delta \mathrm{Ct})$. Primers used for $\mathrm{q}-\mathrm{RT}$-PCR are listed in Supplementary Table 8.

Microscopy and data analysis. CRISPR imaging data were acquired on a Nikon Ti-E inverted wide-field fluorescence microscope equipped with a $\times 100$ NA 1.40 PlanApo oil immersion objective, an LED light source (Excelitas X-Cite XLED1), an sCMOS camera (Hamamatsu Flash 4.0), and a motorized stage (ASI) with stage incubator (Tokai Hit). Acquisitions were controlled by MicroManager. All images were taken as z stacks at $0.4 \mu \mathrm{m}$ steps and with a total of 15 steps and were projected in maximum intensity. Long-term live cell imaging was performed on Andor Dragonfly (high-speed confocal microscopy) based on Nikon TI microscope with Nikon Perfect Focus system, $\times 60$ NA 1.40 objective, an Andor iXon Ultra 888 EM-CCD camera. Images were taken as z stacks at $0.5 \mu \mathrm{m}$ steps (7 steps) and at a frame rate of $5 \mathrm{~Hz}$. Interval time was set as $10 \mathrm{~min}$. Cells were imaged for $4-6 \mathrm{~h}$. 
During image acquisition, cells ware maintained at constant temperature of $37^{\circ} \mathrm{C}$ and $5 \% \mathrm{CO}_{2}$ within an incubation box. All the fluorescence imaging data were analyzed by Image J. Signal-to-noise ratio was defined as the ratio of the intensity of a fluorescent signal and the power of background noise as following formula:

$$
\mathrm{SNR}=\frac{P_{\text {signal }}}{P_{\text {noise }}}=\frac{\text { Max intensity of GFP spot }- \text { Mean intensity of background GFP }}{\text { Std. dev. of background signal }}
$$

\section{Data availability}

The authors declare that all the data supporting the findings of this study are available from the authors on reasonable request.

Received: 26 April 2018 Accepted: 1 November 2018

Published online: 29 November 2018

\section{References}

1. Pombo, A. \& Dillon, N. Three-dimensional genome architecture: players and mechanisms. Nat. Rev. Mol. Cell Biol. 16, 245-257 (2015).

2. Shachar, S. \& Misteli, T. Causes and consequences of nuclear gene positioning. J. Cell Sci. 130, 1501-1508 (2017).

3. Jinek, M. et al. A programmable dual-RNA-guided DNA endonuclease in adaptive bacterial immunity. Science 337, 816-821 (2012).

4. Cong, L. et al. Multiplex genome engineering using CRISPR/Cas systems. Science 339, 819-823 (2013)

5. Mali, P. et al. RNA-guided human genome engineering via Cas9. Science 339, 823-826 (2013).

6. Chen, B. et al. Dynamic imaging of genomic loci in living human cells by an optimized CRISPR/Cas system. Cell 155, 1479-1491 (2013).

7. Chen, B. \& Huang, B. Imaging specific genomic DNA in living cells. Annu. Rev. Biophys. 45, 1-23 (2016).

8. Gu, B. et al. Transcription-coupled changes in nuclear mobility of mammalian cis-regulatory elements. Science 359, 1050-1055 (2018).

9. Qin, P. et al. Live cell imaging of low- and non-repetitive chromosome loci using CRISPR-Cas9. Nat. Commun. 8, 14725 (2017).

10. Maass, P. G., Barutcu, A. R., Shechner, D. M., Weiner, C. L. \& Rinn, J. L. Spatiotemporal allele organization by allele-specific CRISPR live-cell imaging (SNP-CLING). Nat. Struct. Mol. Biol. 25, 176-184 (2017).

11. Hong, Y., Lu, G., Duan, J., Liu, W. \& Zhang, Y. Comparison and optimization of CRISPR/dCas9/gRNA genome-labeling systems for live cell imaging. Genome Biol. 19, 39 (2018)

12. Doench, J. G. et al. Rational design of highly active sgRNAs for CRISPR-Cas9mediated gene inactivation. Nat. Biotechnol. 32, 1262-1267 (2014).

13. Robinett, C. C. et al. In vivo localization of DNA sequences and visualization of large-scale chromatin organization using lac operator/repressor recognition. J. Cell Biol. 135, 1685-1700 1111 (1996).

14. Roukos, V. et al. Spatial dynamics of chromosome translocations in living cells. Science 341, 660-664 (2013).

15. Friedland, A. E. et al. Heritable genome editing in C. elegans via a CRISPR Cas9 system. Nat. Methods 10, 741-743 (2013).

16. Kim, H. et al. A co-CRISPR strategy for efficient genome editing in Caenorhabditis elegans. Genetics 197, 1069-80 (2014).

17. Farboud, B. \& Meyer, B. J. Dramatic enhancement of genome editing by CRISPR/Cas9 through improved guide RNA design. Genetics 199, 959-971 (2015).

18. EI Mouridi, S. et al. Reliable CRISPR/Cas9 genome engineering in Caenorhabditis elegans using a single efficient sgRNA and easily recognizable phenotype. G3 (Bethesda) 7, 1429-1437 (2017).

19. Graham, D. B. \& Root, D. E. Resources for the design of CRISPR gene editing experiments. Genome Biol. 16, 260 (2015).

20. Kamiyama, D. et al. Versatile protein tagging in cells with split fluorescent protein. Nat. Commun. 7, 11046 (2016).

21. Lin, S., Staahl, B. T., Alla, R. K. \& Doudna, J. A. Enhanced homology-directed human genome engineering by controlled timing of CRISPR/Cas9 delivery. eLife 3, e04766 (2014).

22. Zhang, J. P. et al. Efficient precise knockin with a double cut HDR donor after CRISPR/Cas9-mediated double-stranded DNA cleavage. Genome Biol. 18, 35 (2017).

23. Luger, K., Mäder, A. W., Richmond, R. K., Sargent, D. F. \& Richmond, T. J. Crystal structure of the nucleosome core particle at 2.8 A resolution. Nature 389, 251-260 (1997).

24. Germier, T. et al. Real-time imaging of a single gene reveals transcriptioninitiated local confinement. Biophys. J. 113, 1383-1394 (2017).
25. Tasan, I. et al. CRISPR/Cas9-mediated knock-in of an optimized TetO repeat for live cell imaging of endogenous loci. Nucleic Acids Res. 46, e100 (2018).

26. Yao, X. et al. Tild-CRISPR allows for efficient and precise gene knockin in mouse and human cells. Dev. Cell 45, 526-536 (2018).

27. Suzuki, K. et al. In vivo genome editing via CRISPR/Cas9 mediated homologyindependent targeted integration. Nature 540, 144-149 (2016).

28. Sternberg, S. H., Redding, S., Jinek, M., Greene, E. C. \& Doudna, J. A. DNA interrogation by the CRISPR RNA-guided endonuclease Cas9. Nature 507, 62-67 (2014).

29. Jiang, F. et al. Structure of a CRISPR-Cas9 R-loop complex primed for DNA cleavage. Science 351, 867-871 (2016).

30. Gong, S., Yu, H. H., Johnson, K. A. \& Taylor, D. W. DNA unwinding is the primary determinant of CRISPR-Cas9 activity. Cell Rep. 22, 359-371 (2018)

31. Chen, B. et al. Expanding the CRISPR imaging toolset with Staphylococcus aureus Cas9 for simultaneous imaging of multiple genomic loci. Nucleic Acids Res. 44, e75 (2016).

32. Ma, H. et al. Multiplexed labeling of genomic loci with dCas9 and engineered sgRNAs using CRISPRainbow. Nat. Biotechnol. 34, 528-530 (2016).

33. Shao, S. et al. Long-term dual-color tracking of genomic loci by modified sgRNAs of the CRISPR/Cas9 system. Nucleic Acids Res. 44, e86 (2016).

34. Tanenbaum, M. E., Gilbert, L. A., Qi, L. S., Weissman, J. S. \& Vale, R. D. A protein-tagging system for signal amplification in gene expression and fluorescence imaging. Cell 159, 636-646 (2014).

35. Feng, S., Sekine, V., Pessino, H., Li, M. D., Leonetti, B. \& Huang, B. Improved split fluorescent proteins for endogenous protein labeling. Nat. Commun. 8 370 (2017).

36. Chen, B. \& Huang, B. Imaging genomic elements in living cells using CRISPR/ Cas9. Methods Enzymol. 546, 337-354 (2014).

37. Leonetti, M. D., Sekine, S., Kamiyama, D., Weissman, J. S. \& Huang, B. A scalable strategy for high-throughput GFP tagging of endogenous human proteins. Proc. Natl Acad. Sci. USA 113, E3501-E3508 (2016).

\section{Acknowledgements}

We thank D. Kamiyama, S. Sekine, M. Leonetti, S. Feng, and Y. Wang for fruitful discussions. The studies in Chen lab are supported by The Thousand Talent Plan Startup and the National Natural Science Foundation of China (31872819). The research in Huang lab is supported by the W.M. Keck Foundation, the NIH R21EB021453, the NIH Single Cell Analysis Program (R33EB019784 to B.C. and B.H.) and the NIH Extracellular RNA Communication Consortium (U19CA179512, to S.B. and B.H.). B.H. is a Chan Zuckerberg Biohub investigator.

\section{Author contributions}

B.C. conceived the project, executed experiments, analyzed data, and wrote the manuscript. W.Z. deigned the experiments and wrote the manuscript. H.X. and Y.L. performed experiments. B.H. supervised the project and wrote the manuscript.

\section{Additional information}

Supplementary Information accompanies this paper at https://doi.org/10.1038/s41467018-07498-y.

Competing interests: The authors declare no competing interests.

Reprints and permission information is available online at http://npg.nature.com/ reprintsandpermissions/

Publisher's note: Springer Nature remains neutral with regard to jurisdictional claims in published maps and institutional affiliations.

Open Access This article is licensed under a Creative Common Attribution 4.0 International License, which permits use, sharing, adaptation, distribution and reproduction in any medium or format, as long as you give appropriate credit to the original author(s) and the source, provide a link to the Creative Commons license, and indicate if changes were made. The images or other third party material in this article are included in the article's Creative Commons license, unless indicated otherwise in a credit line to the material. If material is not included in the article's Creative Commons license and your intended use is not permitted by statutory regulation or exceeds the permitted use, you will need to obtain permission directly from the copyright holder. To view a copy of this license, visit http://creativecommons.org/ licenses/by/4.0/

(C) The Author(s) 2018 